\title{
Emerging technologies for improving embryo selection: a systematic review
}

\author{
This article was published in the following Dove Press journal: \\ Advanced Health Care Technologies \\ 16 December 2015 \\ Number of times this article has been viewed
}

\author{
Yasemin Sengul' \\ Ayse Bener ${ }^{2}$ \\ Asli Uyar ${ }^{3}$ \\ 'Computer Programming Program, \\ Advanced Vocational Studies, \\ Dogus University, Acibadem, \\ Kadikoy, Istanbul, Turkey; ${ }^{2}$ Data \\ Science Laboratory, Department of \\ Mechanical and Industrial Engineering, \\ Ryerson University, Toronto, ON, \\ Canada; ${ }^{3}$ Department of Computer \\ Engineering, Okan University, Tuzla, \\ Istanbul, Turkey
}

Background: Embryo selection procedure is one of the critical success factors in in vitro fertilization treatment. Various embryo selection technologies have emerged within the past decade. These technologies are either used in combination with morphology or introduced to replace the conventional morphological evaluation. This review aims at investigating the effect of these novel embryo selection technologies on in vitro fertilization success rates.

Methods: A systematic review of the literature was performed among full-text English articles in the PubMed database. Study selection was based on the predefined inclusion and exclusion criteria. Clinical effectiveness of the selected studies was measured in terms of implantation, pregnancy, live birth, and multiple pregnancy rates.

Results: Five studies were identified that fitted the inclusion criteria. In these studies, researchers used aneuploidy screening, metabolomic profiling, and time-lapse imaging analysis as the new technologies. Among these studies, the one that conducted a randomized controlled trial of a commercial time-lapse imaging system demonstrated significant improvement in implantation rate.

Conclusion: Studies using emerging technologies for embryo assessment provide promising results in retrospective analysis. On the other hand, randomized controlled trial studies that test the efficacy of novel embryo selection techniques in clinical practice failed to demonstrate a consistent improvement in the resulting success rates. This review provides a snapshot of the most recent literature on embryo assessment and embryo selection studies. Our findings show that there is a lack of comparative measurements and analyses that are able to assess benefits of the novel technologies in the field of embryo selection.

Keywords: embryo selection, in vitro fertilization, IVF

\section{Introduction}

In vitro fertilization (IVF) is a common assisted reproductive technology used to overcome a range of male or female, or combined factor fertility problems. ${ }^{1}$ The treatment begins with a hormonal therapy to stimulate the development of multiple follicles in the ovary. When follicular maturity is achieved, the oocytes are retrieved and inseminated by sperm under laboratory conditions. Fertilized oocytes are cultured into early embryos for between 2-6 days in special medical equipment. Early embryo development is observed and recorded during the in vitro culture period. Selected embryo(s) are then placed in the woman's uterus, and implantation outcome following the embryo transfer is observed at approximately the 12 th week. A positive implantation outcome is defined as ultrasound-detected fetal cardiac activity at the time of observation.

The success of IVF treatment is significantly affected by the embryo selection procedure. During this procedure, potentially most viable embryo(s) are selected from
Department of Computer Engineering, Okan University, 34959, Tuzla, Istanbul, Turkey

Email asli.uyar@okan.edu.tr (c) (i) (9) 2015 Sengul et al. This work is published by Dove Medical Press Limited, and licensed under Creative Commons Attribution - Non Commercial (unported, v3.0) License. The full terms of the License are available at http://creativecommons.org/licenses/by-nc/3.0/. Non-commercial uses of the work are permitted without any further permission how to request permission may be found at: http://www.dovepress.com/permissions.php 
a cohort of available embryos for subsequent uterine transfer. Transfer of multiple embryos increases the probability of positive pregnancy outcome while also increasing the risk of multiple gestations in IVF treatment. Multiple pregnancies are considered a complication of assisted reproductive technologies, which are associated with poorer maternal and perinatal outcomes $^{2}$ and increased treatment costs. ${ }^{3,4}$ Consequently, there is a worldwide trend to reduce the number of embryos transferred in IVF treatment..$^{5}$ Therefore, embryo selection is a critical step in IVF procedure where identification of a single embryo with the highest reproductive potential is a major challenge for embryologists.

Assessment of embryos by morphological characteristics has been a useful tool since the early days of IVF. In clinical procedure, embryos are transferred either at cleavage stage on day 2 or day 3 or at blastocyst stage on day 5 or day 6 following intra cytoplasmic sperm injection. In classical methods, morphological features are inspected and evaluated at certain time points during preimplantation embryo development. The decision on which embryo to transfer is mainly based on the developmental competence of the embryo on the day of transfer by taking into account morphological observations of that embryo at earlier time points. The scored morphological features include the number of cells, grade of fragmentation, cell size and multinucleation for cleavage stage embryos, and expansion grade and the status of the inner cell mass and the trophectoderm for blastocyst stage embryos. ${ }^{6}$

Morphological assessment of the quality of preimplantation embryos has been associated with increased implantation and pregnancy rates. ${ }^{7}$ On the other hand, as elective single embryo transfers (eSETs) became more prevalent in IVF treatment, embryo selection became more critical and researchers explored new ways to improve the success of selection that is achieved by morphology alone. Within the past decade, novel embryo selection technologies have emerged either to replace morphology-based embryo selection or to enhance conventional morphology-based embryo selection. The aim of this systematic review is to identify the most recent technologies and methods used for embryo assessment and to investigate whether these technologies improve the IVF success rates compared to conventional morphological criteria.

\section{Background}

In this section, we briefly overview the conventional methods and novel technologies used for assessment and selection of oocytes and embryos in IVF laboratories.

\section{Morphological evaluation}

Conventional parameters of embryo developmental competence are based mainly on the static evaluation of the morphology using a light microscope. On the first day following fertilization, pronuclear morphology ${ }^{8}$ and early cleavage ${ }^{9}$ provide information on oocyte-sperm interaction. Scoring on day 2 and day 3 comprises number, size, and symmetry of blastomeres, degree of fragmentation, multinucleation, and appearance of cytoplasm. ${ }^{10,11}$ Morphology-based embryo selection has practical advantages in the clinical routine, and it is associated with significant improvements in implantation and live birth rates. ${ }^{12}$

Morphology-based embryo scoring models are well established and routinely applied in IVF laboratories. On the other hand, researchers continue examining additional morphological parameters to improve the prognostic power of standard embryo scoring systems. ${ }^{13-16}$ For example, Paternot et al investigated the arrangement of blastomeres and showed that tetrahedral four-cell stage embryos on day 2 developed to better quality embryos on day 3 with higher reproductive potential compared with nontetrahedral four-cell stage embryos. ${ }^{14}$ Nevertheless, the precision of morphological criteria is considered less than desired, and the researchers are constantly investigating the viability of additional markers in embryo assessment.

\section{Omics technologies}

Advancements in Omics technologies enabled the investigation of molecular constitution of the embryos and the interaction between the embryo and its surrounding culture media. Such interactions may identify potential nonmorphology markers of embryo viability. Omics research aims to identify what distinct molecules and how many copies of each molecule exist in a biological sample. The DNA, RNA, and protein constitutions of embryos and metabolite content of embryo culture media are investigated by genomics, transcriptomics, proteomics, and metabolomics approaches (reviewed in the study by Uyar and Seli ${ }^{17}$ ).

Preimplantation genetic screening (PGS) is an invasive approach to embryo assessment. It is used to detect chromosomal abnormality by genetic profiling of biopsied blastomeres. PGS primarily tests for aneuploidy where aneuploid embryos are excluded from transfer to reduce the complications associated with genetic abnormalities.

A recent meta-analysis showed that PGS with fluorescence in situ hybridization is associated with lower pregnancy and live birth rates. ${ }^{18}$ On the other hand, PGS using more recent comparative genomic hybridization array or single nucleotide 
polymorphism array technologies has shown promising results in improving pregnancy and live birth rates. ${ }^{19,20}$ In addition, polar body-based aneuploidy has been investigated as a less invasive method; however, resulting implantation rate was not significantly different than the outcome of transfers without aneuploidy screening. ${ }^{21}$

Gene expression occurs by transcription of the DNA into RNAs and subsequent translation of messenger RNAs (mRNAs) into proteins. The term transcriptome refers to the entire set of RNA molecules in a particular cell or tissue, and it can be evaluated as an approximation to the genomewide gene expression. Since follicular cumulus and mural granulosa cells surrounding oocyte reflect the characteristics of the oocyte, ${ }^{22}$ cumulus/granulosa cells transcriptome has been investigated widely as a noninvasive tool to assess oocyte quality as an indicator of embryo viability. ${ }^{23}$ Initial studies reported that transcript levels of candidate genes are associated with embryo quality and clinical outcomes. ${ }^{24-26}$ The most widely studied potential gene markers of oocyte/ embryo quality include $H A S 2$, COX2, GREM1, STAR, AREG, PTGS2, and PTX3.

Alternative noninvasive nonmorphology approaches to embryo assessment are currently based on the analysis of embryo culture media. Proteomics technologies are used to profile the secretome that includes the proteins synthesized by embryos and secreted into the surrounding culture media. Preliminary studies in this context hypothesized that secretome profiles of the culture media could potentially correlate with viability of embryos. Studies using mass spectrometry or protein-array technologies, reported altered expression of specific proteins, such as IL-1 beta, HLA-G, and Ubiquitin, which are associated with blastocyst development or implantation. ${ }^{27-29}$ Although initial studies demonstrated that altered gene expression and secretome profile are associated with embryo viability, the clinical benefit from transcriptomic analysis of cumulus/granulosa cells and proteomic analysis of embryo culture media needs to be demonstrated in randomized controlled trials (RCTs).

Metabolomics-based embryo election techniques profile the changes in metabolite levels in culture media associated with carbohydrate metabolism and amino acid turnover as indicators of normal preimplantation embryo development. ${ }^{30}$ Potential biomarker metabolites identified by various studies include pyruvate, glucose, lactate, glutamate, and asparagine. Specifically, pyruvate uptake on days 1-5 was shown to be associated with embryo and blastocyst development. ${ }^{31-33}$ Also, higher levels of lactate production on days $3-5^{32}$ and glucose uptake on day $4^{33}$ were shown to be correlated with blastocyst development. Besides the carbohydrates, amino acid turnover in the culture media was also investigated as a key component of preimplantation embryo development. Higher levels of asparagine and lower levels of glycine on day 2 were associated with pregnancy and live birth rates. ${ }^{34}$

There is a multitude of retrospective cohort studies reporting significant associations between culture media metabolome and assessed clinical outcomes. ${ }^{35-38}$ On the other hand, a recent meta-analysis showed that the live birth rate is not significantly different when either of the two methods is used in selecting the embryos: 1) near infrared (NIR) spectroscopy-based metabolomic profiling and morphology or 2) morphology alone. ${ }^{39}$ So far, metabolomics has been the most widely investigated noninvasive Omics approach in embryo assessment. However, the efficiency of metabolomics is debated in the literature.

\section{Morphokinetics}

Recent technological advancements led to the development of an instrument consisting of an incubator and a built-in camera for time-lapse imaging of early embryo development in the IVF laboratory. ${ }^{40}$ Time-lapse imaging systems automatically acquire images at predefined time points, and they enable morphokinetic analysis by evaluating the change in embryo morphology over time. Morphokinetic analysis would be a useful tool having the ability to explain the dynamic nature of embryo development better compared to static observations. Initial morphokinetics studies suggest that embryo's developmental potential is correlated with precise timing of specific events such as pronuclear formation, early cleavage, cell division intervals, and initiation of blastulation. ${ }^{41-43}$

\section{Methods}

We designed, conducted, and reported the systematic review based on Moher et al's guidelines called the PRISMA Statement. ${ }^{44}$ First, we defined the research question (RQ) based on the objective of this systematic review. Second, we designed the search strategy to find out the studies and data sources relevant to the RQ. Third, the articles were retrieved through initial database search and the studies were evaluated considering the predefined inclusion and exclusion criteria. Fourth, selected studies went through a detailed quality assessment and evaluation process.

\section{Research question}

This review aims to summarize the studies that report clinical outcomes using novel embryo selection technologies and to 
assess their benefits in the clinical routine. To achieve our goal, we defined the RQ as follows:

RQ: How does the performance of novel embryo selection models compare with conventional morphology-based embryo selection?

RQ aims to conduct comparative analysis of the clinical outcomes reported in the reviewed studies. To extend the systematic review results, we have also discussed the projection of the embryo selection effort on overall IVF success rates by summarizing the publicly available data collected by the Society for Assisted Reproductive Technology (SART); (the authors are not SART members themselves). ${ }^{45}$

\section{Search strategy}

The PubMed electronic database was searched with the keywords "embryo selection" or "embryo assessment" within title, abstract, and keywords of the publications. Since we aim to focus on emerging technologies on embryo selection, the literature search covered the time interval from January 1, 2011 to December 31, 2014, to include the most recent studies in the review.

\section{Study selection}

The publications extracted as a result of literature search have been reviewed according to the following criteria:

- The population: Only clinical (human) studies are included in this review. The study population should be women or couples undergoing IVF treatment.

- The interventions or exposures: Embryo selection or embryo assessment should be conducted by a novel nonmorphology or an adjunct morphology technique.

- The outcomes: Improvement in the implantation and pregnancy rate is the primary validation metric for the success of IVF treatment. Therefore, study should aim to verify the success rate by reviewing clinical outcome in terms of the implantation rate, pregnancy rate, live birth rate, or multiple pregnancy rate. Only the studies reporting one of these outcomes have been included in this review. The study should also include a comparative result between emerging novel technique and conventional morphology-based technique.

- The study design: For the validation of the techniques used, the study should be designed as either a prospective cohort or a randomized control trial.

Study selection performed initially at title level, where reviews, meta-analysis, abstracts, and non-English papers have been excluded from the review. Then, selection criteria applied at abstract level assessment of the articles using the information provided in the abstracts. Finally, candidate full text articles are evaluated in detail considering inclusion and exclusion criteria (defined earlier) to identify the relevant studies, which would provide the potential data for answering the RQ.

\section{Data extraction and quality assessment}

Two of the authors went through the abstracts independently. In the case when the information provided in an abstract did not meet the inclusion criteria, it has been excluded from further review. Otherwise, the paper has been selected as potentially relevant to read the full manuscript. Each paper was assigned randomly to two of the three reviewers. When there was a conflict between the author pairs on a given article, the third author, who was not originally assigned to this paper, reviewed it and the majority voting was taken to include or exclude the study. Similarly, data extraction and quality assessment were independently conducted and cross-checked by two authors. In case of a disagreement, data were finalized with involvement of the third author in the discussion.

\section{Results}

Figure 1 outlines the stages and the results of the search strategy. We first screened the number of publications per year for the period of 2003-2014 as identified through the database search. The trend in the frequency of embryo selection studies varies from 2003 to 2009 , whereas there is a monotonic increase after 2009 (Figure2).

We have included the studies published within a 4-year window, 2011-2014, in our systematic review. A total of 170 relevant publications were extracted at the first phase of the search process. Fourteen studies were excluded from further evaluation after title review. Abstracts of 156 publications have been evaluated as described in the "Methods" section. We have identified 41 publications as potentially relevant to RQs, and we assessed eligibility of these studies for our systematic review. We carefully reviewed full-text articles of the 41 studies and we excluded 36 of them, because they only considered standard morphology-based embryo scoring for embryo selection and the parameters of alternative technique were evaluated retrospectively in relation to cycle outcome. As a result, we identified five publications that meet the eligibility criteria and constitute the final study set for the systematic review.

\section{Effect of novel embryo selection technologies on clinical outcomes}

Selected studies have been investigated in three categories based on the type of the novel embryo selection technique 


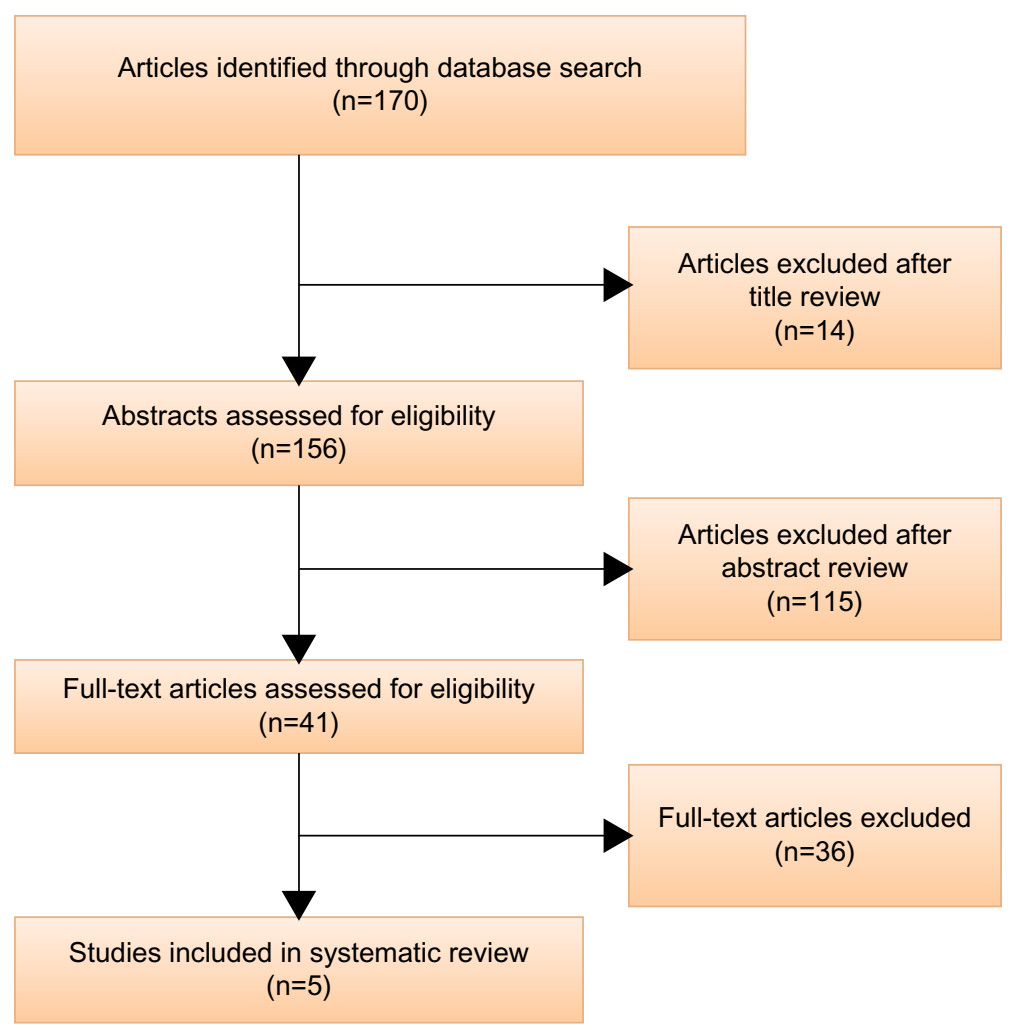

Figure I PRISMA flow diagram of study selection.

used in comparison with conventional morphological evaluation. Table 1 represents characteristics and summary results of the selected studies.

\section{Aneuploidy screening}

This method was used in one of the reviewed studies. ${ }^{46} \mathrm{In}$ an RCT setting, Forman et al tested whether performing eSET after aneuploidy screening of trophectoderm biopsy results in improved obstetric and neonatal outcomes compared with transfer of two untested embryos. ${ }^{46}$ They showed that the delivery rates were similar after euploid eSET $(69 \%)$ and after untested two-embryo transfer (72\%), whereas preterm birth and associated complications were higher when two embryos were transferred without aneuploidy testing. Authors suggested that aneuploidy tested eSETs compromise delivery rates and improve the chance of having a healthy, singleton baby after IVF. We should note that complications related to multiple pregnancies are more likely to occur after transfer of two embryos compared with single embryo transfers.

\section{Metabolomics}

This method is the most commonly studied noninvasive embryo selection technique. Three of the studies we examined use metabolomics-based embryo selection. ${ }^{47-49}$ RCT by
Vergouw et al tested the efficacy of metabolomic profiling of culture NIR spectroscopy and concluded that live birth rate after NIR spectroscopy and morphology (31.7\%) was not significantly different from the live birth rate after embryos were selected by morphology alone $(26.8 \%){ }^{47}$ Hardarson et al compared NIR spectroscopy on spent embryo culture with traditional morphological evaluation, and they found no significant difference in the ongoing pregnancy rate between NIR spectroscopy added to embryo morphology (34.8\%) and morphology alone (35.6\%). ${ }^{48}$ Another RCT by Sfontouris et al also demonstrated that metabolomics analysis does not have a beneficial effect on pregnancy and

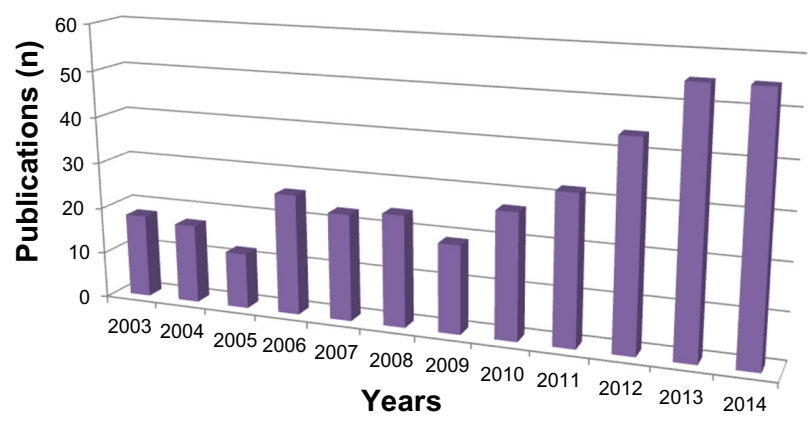

Figure 2 The number of PubMed publications on embryo assessment and embryo selection for each year between 2003 and 2014 . 
Table I Summary of the studies reporting clinical outcomes obtained using a novel embryo selection technique in comparison with morphology alone assessment

\begin{tabular}{|c|c|c|c|c|c|c|}
\hline Study & $\begin{array}{l}\text { Embryo selection } \\
\text { method }\end{array}$ & $\begin{array}{l}\text { Study population } \\
\text { (number of } \\
\text { patients) }\end{array}$ & $\begin{array}{l}\text { Outcome } \\
\text { measure }\end{array}$ & $\begin{array}{l}\text { Outcome } \\
\text { rate - novel } \\
\text { technique } \\
(\%)\end{array}$ & $\begin{array}{l}\text { Outcome rate - } \\
\text { morphology } \\
\text { alone } \\
(\%)\end{array}$ & Results \\
\hline Forman et $\mathrm{al}^{46}$ & $\begin{array}{l}\text { Aneuploidy } \\
\text { screening }\end{array}$ & 175 & $\begin{array}{l}\text { Delivery } \\
\text { rate }\end{array}$ & 69 & 72 & $\begin{array}{l}\text { The delivery rates were similar } \\
\text { ( } 69 \% \text { after euploid eSET vs } 72 \% \\
\text { after untested two-embryo } \\
\text { transfer; } P=0.6) \text { through the } \\
\text { fresh cycle and up to one } \\
\text { frozen transfer, with a dramatic } \\
\text { difference in multiple births } \\
(I .6 \% \text { vs } 47 \% \text {; } P<000 \mathrm{I})\end{array}$ \\
\hline Vergouw et a $\mathrm{a}^{47}$ & Metabolomics & 417 & $\begin{array}{l}\text { Live birth } \\
\text { rate }\end{array}$ & 31.7 & 26.8 & $\begin{array}{l}\text { Day } 3 \text { embryo selection by } \\
\text { metabolomic profiling of culture } \\
\text { medium with NIR spectroscopy } \\
\text { as an addition to morphology } \\
\text { was not able to improve the } \\
\text { ongoing pregnancy and live birth } \\
\text { rates compared with embryo } \\
\text { selection by morphology alone }\end{array}$ \\
\hline Hardarson et al ${ }^{48}$ & Metabolomics & 327 & $\begin{array}{l}\text { Pregnancy } \\
\text { rate }\end{array}$ & 34.8 & 35.6 & $\begin{array}{l}\text { NIR spectroscopy-based } \\
\text { metabolomic profiling of embryo } \\
\text { does not improve the chance } \\
\text { of a viable pregnancy when } \\
\text { performing SET }\end{array}$ \\
\hline Sfontouris et $\mathrm{al}^{49}$ & Metabolomics & 125 & $\begin{array}{l}\text { Implantation } \\
\text { rate }\end{array}$ & 46.8 & 28.9 & $\begin{array}{l}\text { No significant difference for } \\
\text { day } 2 \text { and day } 3 \text { transfers on } \\
\text { implantation rates, where there } \\
\text { is a significant difference on } \\
\text { day } 5 \text { transfers }\end{array}$ \\
\hline Rubio et $\mathrm{al}^{50}$ & Morphokinetic & 843 & $\begin{array}{l}\text { Implantation } \\
\text { rate }\end{array}$ & 44.9 & 37.1 & $\begin{array}{l}\text { Implantation rate was } \\
\text { statistically significantly increased } \\
\text { with morphokinetic evaluation } \\
(44.9 \%) \text { in comparison with } \\
\text { standard morphological } \\
\text { evaluation (37.1\%) }\end{array}$ \\
\hline
\end{tabular}

Abbreviations: eSET, elective single embryo transfer; NIR, near infrared; SET, single embryo transfer.

live birth rates, but it results in improved implantation rate for day 5 transfers (46.8\% vs $28.9 \%){ }^{49}$ This RCT could not provide a solid conclusion since the study was terminated prematurely due to the market withdrawal of commercial metabolomics instrument.

\section{Morphokinetics}

RCT by Rubio et al used EmbryoScope time-lapse monitoring system and showed that implantation rate was statistically significantly increased with morphokinetic evaluation (44.9\%) in comparison with standard morphological evaluation (37.1\%). ${ }^{50}$ Same study also reported significant increase in ongoing pregnancy $(51.4 \%$ vs $41.7 \%)$, significant decrease in early pregnancy loss ( $16.6 \%$ vs $25.8 \%)$, and nonsignificant increase in pregnancy rate $(61.6 \%$ vs $56.3 \%)$ with the use of morphokinetic features for embryo selection.

\section{IVF success rates}

Data presented in this review showed that only a small fraction of embryo selection studies perform comparative analysis of embryo selection techniques in a prospective cohort or an RCT setting, and a few report actual comparative clinical outcomes. To examine the broader implications of embryo selection effort, we analyzed the IVF success rates in USA using the data obtained from the SART Clinical Outcome Reporting System, Clinical Summary Report, which contains yearly information of all IVF cycles performed by reporting clinics. ${ }^{45}$

A total of 1,539,395 cycles with transfer of fresh embryos from nondonor oocytes were reported to SART for the period of 2003-2013 (Figure 3A). Nearly 70\% of all cycles were performed in patients $\geq 35$ years of age. As a result of the effort in favoring eSET, the average number of transferred embryos was dramatically reduced from the range of $2.7-3$ in 
2003 to $1.6-1.9$ in 2013 including all age groups of patients (Figure 3B).

Success of IVF treatment is mainly measured by implantation outcome. Implantation is a complex process involving both the mother and the embryo. Successful embryo implantation depends on female factors, eg, a well-functioning endometrium, as well as embryonic factors. Advancements in the treatment of female infertility enabled better manipulation of endometrial-embryo interaction for successful implantation and addressed various treatments for repeated implantation failures. ${ }^{51}$ Within the period of 2003-2013, implantation rate increased gradually from $27.6 \%$ to $39.5 \%$, $20.8 \%$ to $30.0 \%$, and $13.9 \%$ to $19.3 \%$ for the age groups of $<35,35-37$, and 38-40 years, respectively (Figure 3C). This increase may be attributed to the combined effect of improvements in treatment options for successful implantation and enhanced embryo selection strategies. Reduction in the average number of transferred embryos limited the transfer of low quality embryos and decreased the number of negative implantation outcomes dominantly.
A

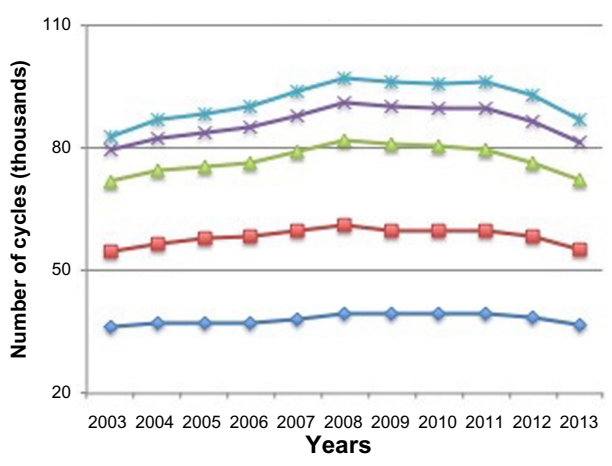

C

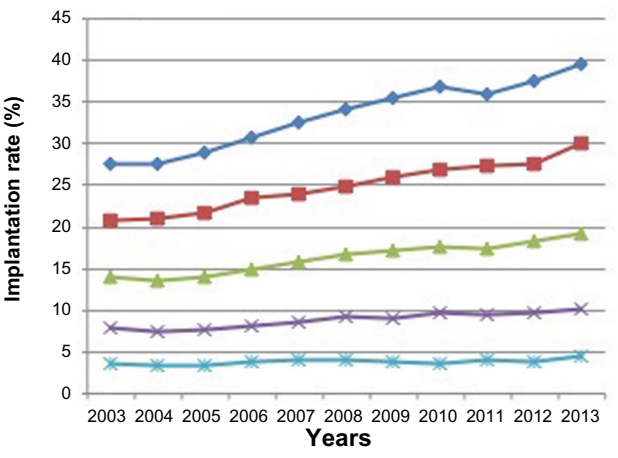

E

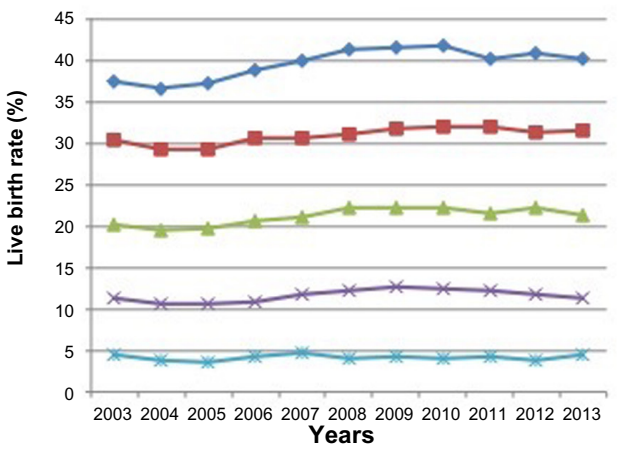

B

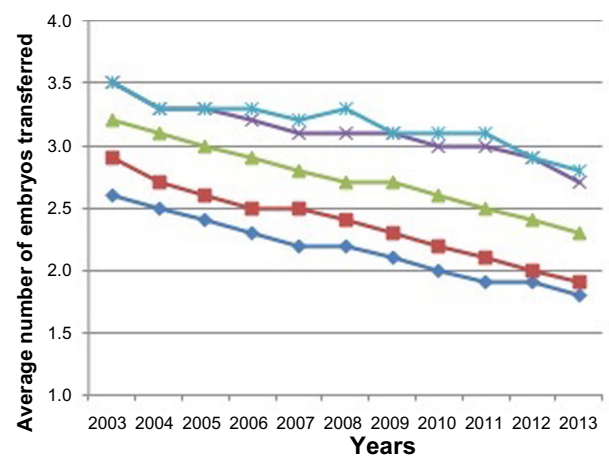

D

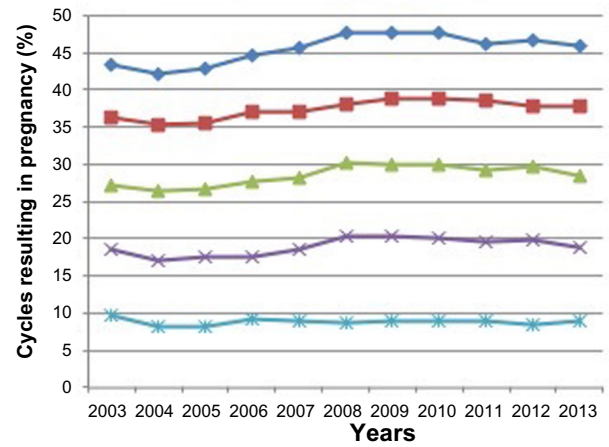

F

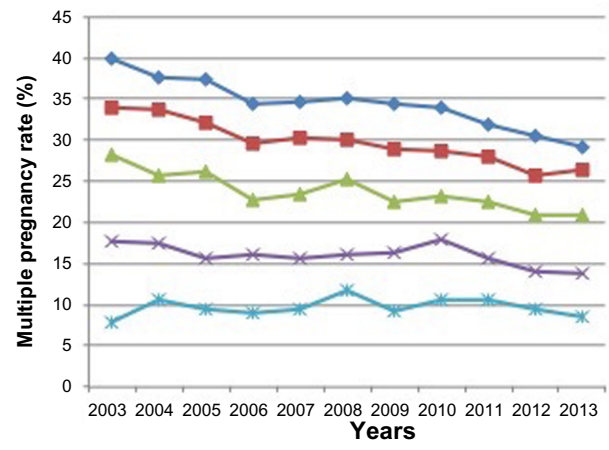

Age categories (years)

$\rightarrow<35 \rightarrow-35-37 \multimap 38-40 \div 41-42 \longleftarrow 42$

Figure 3 IVF success rates obtained online from the Society for Assisted Reproductive Technology (SART) Clinical Outcomes Reporting System, Clinical Summary Report, for the period of 2003-2013.

Notes: Assessed success rates are reported separately for the age groups consistent with SART age categorization. (A) Number of cycles; (B) average number of transferred embryos; (C) implantation rate; (D) pregnancy rate; (E) live birth rate; (F) multiple pregnancy rate. Data from the SART Clinical Outcomes Reporting System, Clinical Summary Report; (C) Copyright 2015 SART, All Rights Reserved. The investigators summarized publicly available data collected by SART, and are not SART members themselves. The data is accessed from the National Summary provided annually from 2004-20I3 on www.sart.org. ${ }^{45}$

Abbreviation: IVF, in vitro fertilization. 
Interestingly, the improvement in implantation rates does not reflect to pregnancy and live birth rates. Pregnancy rates slightly increased with changes $<3 \%$ in patients $\leq 42$ years of age, and slightly decreased from $9.8 \%$ in 2003 to $8.9 \%$ in 2013 in patients $>42$ years of age (Figure 3D). Live birth rates remained the same in patients $>40$ years of age and slightly increased in patients $\leq 40$ years of age (Figure 3E). Pregnancy and live birth rates reached the maximum values in 2010 with $47.7 \%$ and $41.7 \%$, respectively, in the $<35$ years of age group. Consistent with the decrease in average number of embryos transferred, multiple pregnancy rates decreased considerably in patients $\leq 42$ years of age (Figure 3F).

\section{Discussion}

Selecting the right embryo is a very important step in IVF treatment process. Clinicians most routinely use a method based on static morphological analysis to select the highest quality embryos. This systematic review showed that several techniques have been proposed and employed for embryo selection. However, there is no consensus on the clinical benefits of these techniques. Out of 156 studies reviewed, only five of them were identified as eligible for review. Among those, only one of the studies offers an intervention that shows an improvement in pregnancy outcomes. ${ }^{50}$

This review demonstrated that adjunct morphological evaluations were possible using the light microscope in the standard laboratory setting. These evaluations were mostly employed retrospectively. Aneuploidy testing was criticized due to invasiveness, and it is shown to reduce the implantation rates when a biopsied embryo with a negative test result was transferred. On the other hand, the efficacy of genetic screening and diagnosis should be evaluated considering the fact that the genetic testing is unavoidable in cases where the likelihood of chromosomal abnormalities increases with older maternal age, recurrent implantation failures or a family history of genetic disorder.

Further, transcriptomics approach provided a noninvasive means of oocyte and embryo assessment by analysis of gene expression levels in cumulus and granulosa cells and enabled identification of certain gene markers of embryo viability. Metabolomics and proteomics approaches are also noninvasive techniques investigating embryo's interaction with its culture media to identify predictors of reproductive potential. Utilization of metabolomics, transcriptomics, and proteomics approaches for embryo selection is mostly restricted to availability of bench-top instruments or rapid technologies. These instruments and technologies would enable testing of embryo viability within the embryo culture time frame for fresh embryo transfer cycles. Commercial instruments have been developed for metabolomics analysis, and therefore, metabolomics has been the most widely studied Omics approach within the context of IVF. On the other hand, RCTs showed no benefit from metabolomics-based embryo selection compared with morphology alone criteria. Currently, there is no bench-top instrument for genome-wide transcriptomics or proteomics analysis limiting the applicability of these techniques in IVF laboratories.

Development of commercial instruments for time-lapse imaging of embryos during incubation in the laboratory enabled morphokinetic evaluation of embryo development. Recorded morphokinetic parameters were evaluated in relation to cycle outcome to identify potential novel markers. Initial studies showed that embryo selection using morphokinetic markers improve the success rates when compared with the outcomes obtained through standard morphology-based embryo selection. The number of IVF clinics using timelapse imaging systems has been increasing. We may then argue that continuous monitoring of embryo developmental kinetics is likely to replace conventional light microscopebased morphological evaluation at fewer time points during embryo culture period. Also, a recent publication illustrated the development of an algorithm to detect aneuploidy in a noninvasive manner using time-lapse imaging of IVF embryos..$^{52}$ However, some researchers claim that the data presented in that study may be premature..$^{53}$ Nevertheless, the superior prognostic power of morphokinetic parameters needs to be validated in multiple RCTs to provide a more solid conclusion about efficiency of time-lapse embryo imaging systems.

Novel embryo assessment techniques are strongly dependent on bio technological advancements. However, it is not clear whether the predictor value of the data obtained from available technologies is being evaluated adequately. Various sophisticated prediction algorithms have been developed as part of clinical decision support systems to help physicians with the decision of medical diagnosis and evaluation of the treatment options. However, the use of such algorithms is limited in IVF treatment. Recently, a machine learningbased prediction model was proposed to evaluate embryo and cycle characteristics together to provide an implantation probability for individual IVF embryos. ${ }^{54}$ Authors suggested that automated and integrated analysis of predictor features coming from different stages of IVF treatment is likely to improve embryo selection and decision on the number of embryos to transfer. ${ }^{54}$ 


\section{Conclusion}

Embryo selection has been an active research field since the early days of IVF. However, there is no consensus on the best method of embryo selection. New embryo assessment technologies enable identification of new markers of embryo quality. These new markers are analyzed in retrospective study designs in relation to treatment outcome. Then, the true prognostic value of the novel technique is tested in RCTs. A novel technique would survive only if it succeeds the RCT level validation. Further, considering the limited effect of embryo selection effort on overall success rates, it could be speculated that embryo selection is reaching its limits and it is time to focus more on "making the best embryo" rather than "selecting the best embryo".

\section{Disclosure}

The authors report no conflicts of interest in this work.

\section{References}

1. Steptoe PC, Edwards RG. Birth after the reimplantation of a human embryo. Lancet. 1978;2:366.

2. Black M, Bhattacharya S. Epidemiology of multiple pregnancy and the effect of assisted conception. Semin Fetal Neonatal Med. 2010;15: 306-312.

3. Chambers GM, Ledger W. The economic implications of multiple pregnancy following ART. Semin Fetal Neonatal Med. 2014;19:254-261.

4. Lemos EV, Zhang D, Van Voorhis BJ, Hu XH. Healthcare expenses associated with multiple vs singleton pregnancies in the United States. Am J Obstet Gynecol. 2013;209:e581-e586.

5. Pandian Z, Templeton A, Serour G, Bhattacharya S. Number of embryos for transfer after IVF and ICSI: a cochrane review. Hum Reprod. 2005;20:2681-2687.

6. Lundin K, Ahlstrom A. Quality control and standardization of embryo morphology scoring and viability markers. Reprod Biomed Online. 2015;31(4):459-471.

7. Toner JP. Progress we can be proud of: US trends in assisted reproduction over the first 20 years. Fertil Steril. 2002;78:943-950.

8. Scott L. Pronuclear scoring as a predictor of embryo development. Reprod Biomed Online. 2003;6:201-214.

9. Ciray HN, Karagenç L, Ulug U, Bener F, Bahçeci M. Early cleavage morphology affects the quality and implantation potential of day 3 embryos. Fertil Steril. 2006;85:358-365.

10. Cummins JM, Breen TM, Harrison KL, Shaw JM, Wilson LM, Hennessey JF. A formula for scoring human embryo growth rates in in vitro fertilization: its value in predicting pregnancy and in comparison with visual estimates of embryo quality. J In Vitro Fert Embryo Transf. 1986;3:284-295.

11. Puissant F, Van Rysselberge M, Barlow P, Deweze J, Leroy F. Embryo scoring as a prognostic tool in IVF treatment. Hum Reprod. 1987;2: 705-708.

12. Dennis SJ, Thomas MA, Williams DB, Robins JC. Embryo morphology score on day 3 is predictive of implantation and live birth rates. $J$ Assist Reprod Genet. 2006;23:171-175.

13. Sela R, Samuelov L, Almog B, et al. An embryo cleavage pattern based on the relative blastomere size as a function of cell number for predicting implantation outcome. Fertil Steril. 2012;98(650-656):e654.

14. Paternot G, Debrock S, De Neubourg D, D’Hooghe TM, Spiessens C The spatial arrangement of blastomeres at the 4-cell stage and IVF outcome. Reprod Biomed Online. 2014;28:198-203.
15. Nicoli A, Capodanno F, Rondini I, et al. Pronuclear morphology evaluation in in vitro fertilization (IVF)/intracytoplasmic sperm injection (ICSI) cycles: a retrospective clinical review. J Ovarian Res. 2013;6:1.

16. Berger DS, Zapantis A, Merhi Z, Younger J, Polotsky AJ, Jindal SK. Embryo quality but not pronuclear score is associated with clinical pregnancy following IVF. J Assist Reprod Genet. 2014;31:279-283.

17. Uyar A, Seli E. Noninvasive techniques: embryo selection by transcriptomics, proteomics, and metabolomics. In: Montag M, editor. A Practical Guide to Selecting Gametes and Embryos. Boca Raton, FL: CRC Press; 2014:191-208.

18. Checa MA, Alonso-Coello P, Sola I, et al. IVF/ICSI with or without preimplantation genetic screening for aneuploidy in couples without genetic disorders: a systematic review and meta-analysis. J Assist Reprod Genet. 2009;26:273-283.

19. Sher G, Keskintepe L, Keskintepe M, Maassarani G, Tortoriello D, Brody S. Genetic analysis of human embryos by metaphase comparative genomic hybridization (mCGH) improves efficiency of IVF by increasing embryo implantation rate and reducing multiple pregnancies and spontaneous miscarriages. Fertil Steril. 2009;92:1886-1894.

20. Schoolcraft WB, Treff NR, Stevens JM, Ferry K, Katz-Jaffe M, Scott RT Jr. Live birth outcome with trophectoderm biopsy, blastocyst vitrification, and single-nucleotide polymorphism microarray-based comprehensive chromosome screening in infertile patients. Fertil Steril. 2011;96:638-640.

21. Salvaggio CN, Forman EJ, Garnsey HM, Treff NR, Scott RT Jr. Polar body based aneuploidy screening is poorly predictive of embryo ploidy and reproductive potential. J Assist Reprod Genet. 2014;31:1221-1226.

22. Tanghe S, Van Soom A, Nauwynck H, Coryn M, de Kruif A. Minireview: functions of the cumulus oophorus during oocyte maturation, ovulation, and fertilization. Mol Reprod Dev. 2002;61:414-424.

23. Uyar A, Torrealday S, Seli E. Cumulus and granulosa cell markers of oocyte and embryo quality. Fertil Steril. 2013;99:979-997.

24. Cillo F, Brevini TA, Antonini S, Paffoni A, Ragni G, Gandolfi F. Association between human oocyte developmental competence and expression levels of some cumulus genes. Reproduction. 2007;134: 645-650.

25. Assou S, Haouzi D, Mahmoud K, et al. A non-invasive test for assessing embryo potential by gene expression profiles of human cumulus cells: a proof of concept study. Mol Hum Reprod. 2008;14:711-719.

26. Hamel M, Dufort I, Robert C, Léveillé MC, Leader A, Sirard MA. Genomic assessment of follicular marker genes as pregnancy predictors for human IVF. Mol Hum Reprod. 2010;16:87-96.

27. Katz-Jaffe MG, Schoolcraft WB, Gardner DK. Analysis of protein expression (secretome) by human and mouse preimplantation embryos. Fertil Steril. 2006;86:678-685.

28. Domínguez F, Gadea B, Esteban FJ, Horcajadas JA, Pellicer A, Simón C. Comparative protein-profile analysis of implanted versus non-implanted human blastocysts. Hum Reprod. 2008;23:1993-2000.

29. Dominguez F, Gadea B, Mercader A, Esteban FJ, Pellicer A, Simón C. Embryologic outcome and secretome profile of implanted blastocysts obtained after coculture in human endometrial epithelial cells versus the sequential system. Fertil Steril. 2010;93(774-782):e771.

30. Uyar A, Seli E. Metabolomic assessment of embryo viability. Semin Reprod Med. 2014;32:141-152.

31. Hardy K, Hooper MA, Handyside AH, Rutherford AJ, Winston RM, Leese HJ. Non-invasive measurement of glucose and pyruvate uptake by individual human oocytes and preimplantation embryos. Hum Reprod. 1989;4:188-191.

32. Gott AL, Hardy K, Winston RM, Leese HJ. Non-invasive measurement of pyruvate and glucose uptake and lactate production by single human preimplantation embryos. Hum Reprod. 1990;5:104-108.

33. Gardner DK, Lane M, Stevens J, Schoolcraft WB. Noninvasive assessment of human embryo nutrient consumption as a measure of developmental potential. Fertil Steril. 2001;76:1175-1180.

34. Brison DR, Houghton FD, Falconer D, et al. Identification of viable embryos in IVF by non-invasive measurement of amino acid turnover. Hum Reprod. 2004;19:2319-2324. 
35. Seli E, Sakkas D, Scott R, Kwok SC, Rosendahl SM, Burns DH. Noninvasive metabolomic profiling of embryo culture media using Raman and near-infrared spectroscopy correlates with reproductive potential of embryos in women undergoing in vitro fertilization. Fertil Steril. 2007;88:1350-1357.

36. Scott R, Seli E, Miller K, Sakkas D, Scott K, Burns DH. Noninvasive metabolomic profiling of human embryo culture media using Raman spectroscopy predicts embryonic reproductive potential: a prospective blinded pilot study. Fertil Steril. 2008;90:77-83.

37. Vergouw CG, Botros LL, Roos P, et al. Metabolomic profiling by near-infrared spectroscopy as a tool to assess embryo viability: a novel, non-invasive method for embryo selection. Hum Reprod. 2008;23: 1499-1504.

38. Seli E, Vergouw CG, Morita H, et al. Noninvasive metabolomic profiling as an adjunct to morphology for noninvasive embryo assessment in women undergoing single embryo transfer. Fertil Steril. 2010;94: $535-542$.

39. Vergouw CG, Heymans MW, Hardarson T, et al. No evidence that embryo selection by near-infrared spectroscopy in addition to morphology is able to improve live birth rates: results from an individual patient data meta-analysis. Hum Reprod. 2014;29:455-461.

40. Meseguer M, Herrero J, Tejera A, Hilligsøe KM, Ramsing NB, Remohí J. The use of morphokinetics as a predictor of embryo implantation. Hum Reprod. 2011;26:2658-2671.

41. Desai N, Ploskonka S, Goodman LR, Austin C, Goldberg J, Falcone T. Analysis of embryo morphokinetics, multinucleation and cleavage anomalies using continuous time-lapse monitoring in blastocyst transfer cycles. Reprod Biol Endocrinol. 2014;12:54.

42. Dal Canto M, Coticchio G, Mignini Renzini M, et al. Cleavage kinetics analysis of human embryos predicts development to blastocyst and implantation. Reprod Biomed Online. 2012;25:474-480.

43. Kirkegaard K, Kesmodel US, Hindkjaer JJ, Ingerslev HJ. Time-lapse parameters as predictors of blastocyst development and pregnancy outcome in embryos from good prognosis patients: a prospective cohort study. Hum Reprod. 2013;28:2643-2651.

44. Moher D, Liberati A, Tetzlaff J, et al. Preferred reporting items for systematic reviews and meta-analyses: the PRISMA statement. J Clin Epidemiol. 2009;62:1006-1012.
45. Society for Assisted Reproductive Technology, Clinical Outcome Reporting System, Clinical Summary Report. Available from: https:// www.sartcorsonline.com/rptCSR_PublicMultYear.aspx?ClinicPKID $=0$. Accessed March 11, 2015.

46. Forman EJ, Hong KH, Franasiak JM, Scott RT Jr. Obstetrical and neonatal outcomes from the BEST Trial: single embryo transfer with aneuploidy screening improves outcomes after in vitro fertilization without compromising delivery rates. Am J Obstet Gynecol. 2014; 210(157):e151-e156.

47. Vergouw CG, Kieslinger DC, Kostelijk EH, et al. Day 3 embryo selection by metabolomic profiling of culture medium with near-infrared spectroscopy as an adjunct to morphology: a randomized controlled trial. Hum Reprod. 2012;27:2304-2311.

48. Hardarson T, Ahlström A, Rogberg L, et al. Non-invasive metabolomic profiling of Day 2 and 5 embryo culture medium: a prospective randomized trial. Hum Reprod. 2012;27:89-96.

49. Sfontouris IA, Lainas GT, Sakkas D, Zorzovilis IZ, Petsas GK, Lainas TG. Non-invasive metabolomic analysis using a commercial NIR instrument for embryo selection. J Hum Reprod Sci. 2013;6:133-139.

50. Rubio I, Galán A, Larreategui Z, et al. Clinical validation of embryo culture and selection by morphokinetic analysis: a randomized, controlled trial of the EmbryoScope. Fertil Steril. 2014;102(1287-1294):e1285.

51. Simon A, Laufer N. Repeated implantation failure: clinical approach. Fertil Steril. 2012;97:1039-1043.

52. Campbell A, Fishel S, Bowman N, Duffy S, Sedler M, Thornton S. Retrospective analysis of outcomes after IVF using an aneuploidy risk model derived from time-lapse imaging without PGS. Reprod Biomed Online. 2013;27:140-146.

53. Ottolini C, Rienzi L, Capalbo A. A cautionary note against embryo aneuploidy risk assessment using time-lapse imaging. Reprod Biomed Online. 2014;28:273-275.

54. Uyar A, Bener A, Ciray HN. Predictive modeling of implantation outcome in an in vitro fertilization setting: an application of machine learning methods. Med Decis Making. Epub 2014 May 19.
Advanced Health Care Technologies

\section{Publish your work in this journal}

Advanced Health Care Technologies is an international, peer reviewed, open access journal that provides a unique forum for articles on: point-of-care, health care diagnostics and treatment, bioengineering, biotechnology, biosensing, electronics, clinical/medical science, chemical engineering, materials science, regenerative medicine, micro-/

\section{Dovepress}

nano-technologies, and methods and applications for nanoscience and nanotechnology. The manuscript management system is completely online and includes a very quick and fair peer review system, which is all easy to use. Visit http://www.dovepress.com/testimonials.php to read real quotes from published authors. 